\title{
IMPULSIVE INTEGRAL EQUATIONS IN BANACH SPACES AND APPLICATIONS ${ }^{1}$
}

\author{
DAJUN GUO \\ Department of Mathematics \\ Shandong University \\ Jinan, PEOPLE'S REPUBLIC OF CHINA
}

\begin{abstract}
In this paper, we first use the fixed point theory to prove two existence theorems of positive solutions for the impulsive Fredholm integral Equations in Banach spaces. And then, we offer some applications to the two-point boundary value problems for the second order impulsive differential equations in Banach spaces.
\end{abstract}

Key words: Impulsive Fredholm integral equation, impulsive differential equation, strict set contraction, fixed point theorem.

AMS (MOS) subject classification: $\quad 45 \mathrm{~N} 05,47 \mathrm{H} 07$.

\section{INTRODUCTION}

The theory of impulsive differential equations is a new important branch of differential equations (see [5]). Of course, impulsive integral equations and impulsive differential equations are closely connected. Paper [3] discussed the impulsive Volterra integral equations in Banach spaces based on the iterative monotone technique and a comparison result. Since there is no comparison theorem for impulsive Fredholm integral equations, the method in [3] is not available for such equations. In this paper, we first use the fixed point theory to prove two existence theorems of positive solutions for impulsive Fredholm integral equations in Banach spaces. And then, we offer some applications to the two-point boundary value problems for second order impulsive differential equations in Banach spaces.

Let the real Banach space $E$ be partially ordered by a cone $P$ of $E$, i.e., $x \leq y$ iff $y-x \in P . \quad P$ is said to be normal if there exists a positive constant $N$ such that $\theta \leq x \leq y$ implies $\|x\| \leq N\|y\|$, where $\theta$ denotes the zero element of $E$, and $N$ is called the normal constant of $P$ (see [4]). Consider the impulsive Fredholm integral equation in $E$ :

\footnotetext{
${ }^{1}$ Received: February, 1991. Revised: April, 1991.
} 


$$
x(t)=\int_{0}^{T} H(t, s, x(s)) d s+\sum_{0<t_{k}<t} I_{k}\left(x\left(t_{k}\right)\right),
$$

where $\quad H \in C[J \times J \times P, P], J=[0, T], \quad I_{k} \in C[P, P] \quad(k=1,2, \ldots, p) \quad$ and $0<t_{1}<\ldots<t_{k} \ldots<t_{p}<T$. Assume that $H(t, s, \theta)=\theta$ for $t, s \in J$ and $I_{k}(\theta)=\theta$ $(k=1,2, \ldots, p)$, then $x(t) \equiv \theta$ is the trivial solution of equation (1). Let $P C[J, E]=\{x: x$ is a map from $J$ into $E$ such that $x(t)$ is continuous at $t \neq t_{k}$, left continuous at $t=t_{k}$ and $\lim _{t \rightarrow t_{k}^{+}} x(t)$ exist for $\left.k=1,2, \ldots, p\right\}$. Evidently, $P C[J, E]$ is a Banach space with norm $\|x\|_{p}=\sup _{t \in J}\|x(t)\|$. Clearly, $Q=\{x \in P C[J, E]: x(t) \geq \theta$ for $t \in J\}$ is a normal cone in space $P C[J, E]$ if $P$ is normal. A map $x \in P C[J, E]$ is called a positive solution of equation (1) if it satisfies (1) on $J$ and $x \in Q, x \neq \theta$.

\section{LEMMAS}

Let $J_{0}=\left[0, t_{1}\right], J_{1}=\left(t_{1}, t_{2}\right], \ldots, J_{p-1}=\left(t_{p-1}, t_{p}\right], J_{p}=\left(t_{p}, T\right] . \quad$ For $S \subset P C[J, E]$, we denote $S(t)=\{x(t): x \in S\} \subset E(t \in J)$.

Lemma 1: If $S$ is bounded and the elements of $S$ are equicontinuous on each $J_{k}(k=0,1, \ldots, p)$, then

$$
\alpha(S)=\sup _{t \in J} \alpha(S(t))
$$

where $\alpha$ denotes the Kuratowski measure of noncompactness.

Proof: Let $S^{(k)}=\left\{\left.x\right|_{J_{k}}: x \in S\right\}(k=0,1, \ldots, p)$. Since $\lim _{t \rightarrow t} \underset{k}{ } x(t)$ exists, $S^{(k)}$ may be regarded as a subset of the space $C\left[\bar{J}_{k}, E\right]$, where $\bar{J}_{k}$ is the closure of $J_{k}$, i.e., $\bar{J}_{k}=\left[t_{k-1}, t_{k}\right]$. Hence (see [6])

$$
\alpha\left(S^{(k)}\right)=\sup _{t \in J_{k}} \alpha\left(S^{(k)}(t)\right)(k=0,1, \ldots, p)
$$

Obviously, $S(t)=S^{(k)}(t)$ for $t \in J_{k}, k=0,1, \ldots, p$, so

$$
\sup _{t \in J} \alpha(S(t))=\max _{k} \sup _{t \in J_{k}} \alpha\left(S^{(k)}(t)\right)
$$

Now, we show

$$
\alpha(S)=\max _{k} \alpha\left(S^{(k)}\right) .
$$

It is evident,

$$
\alpha\left(S^{(k)}\right) \leq \alpha(S)(k=0,1, \ldots, p)
$$


On the other hand, for any given $\epsilon>0$, there exists partition $S^{(k)}=\bigcup_{i=1}^{n} S_{i}{ }^{(k)}$ such that

$$
\operatorname{diam}\left(S_{i}{ }^{(k)}\right)<\alpha\left(S^{(k)}\right)+\epsilon \leq b+\epsilon \quad\left(i=1,2, \ldots, n_{k}\right),
$$

where $b=\max _{k} \alpha\left(S^{(k)}\right)$. Let $S_{i}^{(k)}=\left.S_{k i}\right|_{J_{k}}$ and $S(i, j, \ldots, h)=S_{1 i} \cap S_{2 j} \cap \ldots \cap S_{p h}$, then $S=\cup\left\{S(i, j, \ldots, h): i=1,2, \ldots, n_{1} ; j=1,2, \ldots, n_{2} ; \ldots ; h=1,2, \ldots, n_{p}\right\}$, and by $(7)$,

$$
\operatorname{dim}(S(i, j, \ldots, h)) \leq b+\epsilon\left(i=1,2, \ldots, n_{1} ; j=1,2, \ldots, n_{2} ; \ldots ; h=1,2, \ldots, n_{p}\right) .
$$

Hence

$$
\alpha(S) \leq b+\epsilon
$$

which implies, since $\epsilon$ is arbitrary, that

$$
\alpha(S) \leq b
$$

Consequently, (5) follows from (6) and (8), and finally, (2) follows from (3), (4) and (5). The proof is complete.

In the following, the closed balls in $E$ and $P C[J, E]$ are denoted by $T_{r}=\{x \in E:\|x\| \leq r\}$ and $B_{r}=\left\{x \in P C[J, E]:\|x\|_{p} \leq r\right\}$ respectively.

Consider the operator

$$
A x(t)=\int_{0}^{T} H(t, s, x(s)) d s+\sum_{0<t_{k}<t} I_{k}\left(x\left(t_{k}\right)\right) .
$$

Lemma 2: Let $H \in C[J \times J \times P, P]$ and $I_{k} \in C[P, P](k=1,2, \ldots, p)$. Suppose that, for any $r>0, H$ is uniformly continuous on $J \times J \times\left(P \cap T_{r}\right), I_{k}$ is bounded on $P \cap T_{r}$, and there exist nonnegative constants $L_{r}$ and $M_{r}{ }^{(k)}$ with

such that

$$
2 T L_{r}+\sum_{k=1}^{p} M_{r}^{(k)}<1
$$

$$
\alpha(H(t, s, D)) \leq L_{r} \alpha(D), \quad t, s \in J, \quad D \subset P \cap T_{r}
$$

and

$$
\alpha\left(I_{k}(D)\right) \leq M_{r}^{(k)} \alpha(D), \quad D \subset P \cap T_{r}, \quad k=1,2, \ldots, p .
$$

Then, for any $r>0$, operator $A$ is a strict set contraction from $Q \cap B_{r}$ into $Q$, i.e., there exists a constant $k_{r}$ with $0 \leq k_{r}<1$ such that $\alpha(A(S)) \leq k_{r} \alpha(S)$ for any $S \subset Q \cap B_{r}$. 
Proof: It is easy to see that the uniform continuity of $H$ on $J \times J \times\left(P \cap T_{r}\right)$ implies the boundedness of $H$ on $J \times J \times\left(P \cap T_{r}\right)$, and so $A$ is a bounded and continuous operator from $Q \cap B_{r}$ into $Q$. By the uniform continuity of $H$ and (10) and using [6], we have

$$
\alpha(H(J \times J \times D))=\max _{t, s \in J} \alpha(H(t, s, D)) \leq L_{r} \alpha(D), D \subset P \cap T_{r} .
$$

Now, let $S \subset Q \cap B_{r}$ be arbitrarily given. By virtue of (9), it is easy to show that the elements of $A(S)$ are equicontinuous on each $J_{k}(k=0,1, \ldots, p)$, and so, by Lemma 1 ,

$$
\alpha(A(S))=\sup _{t \in J} \alpha(A(S(t)))
$$

Using (12), (11) and the obvious formula

$$
\int_{0}^{T} y(s) d s \in T \overline{c o}\{y(s): s \in J\}, \quad y \in P C[J, E],
$$

we find

$$
\begin{aligned}
\alpha(A(S(t))) \leq T \alpha(\overline{c o}\{H(t, s, x(s)): x \in S, s \in J\})+\sum_{0<t_{k}<t} \alpha\left(\left\{I_{k}\left(x\left(t_{k}\right)\right): x \in S\right\}\right) \\
\leq T \alpha\left(H(J \times J \times S(J))+\sum_{k=1}^{p} \alpha\left(I_{k}\left(S\left(t_{k}\right)\right)\right)\right. \\
\leq T L_{r} \alpha(S(J))+\sum_{k=1}^{p} M_{r}{ }^{(k)} \alpha\left(S\left(t_{k}\right)\right)
\end{aligned}
$$

where $S(J)=\{x(s): x \in S, s \in J\}$ and $S\left(t_{k}\right)=\left\{x\left(t_{k}\right): x \in S\right\}$. For any given $\epsilon>0$, there exists a partition $S=\bigcup_{j=1}^{m} S_{j}$ such that

$$
\operatorname{diam}\left(S_{j}\right)<\alpha(S)+\epsilon, j=1,2, \ldots, m
$$

Since $S\left(t_{k}\right)=\bigcup_{j=1}^{m} S_{j}\left(t_{k}\right)$ and $\operatorname{diam}\left(S_{j}\left(t_{k}\right)\right) \leq \operatorname{diam}\left(S_{j}\right)$, we find by (15),

$$
\alpha\left(S\left(t_{k}\right)\right) \leq \alpha(S)+\epsilon, k=1,2, \ldots, p
$$

On the other hand, choosing $x_{j} \in S_{j}(j=1,2, \ldots, m)$ and a partition $J_{k}=\bigcup_{i=1}^{n} J_{k}{ }^{(i)}$ $(k=0,1,2, \ldots, p)$ such that

$$
\left\|x_{j}(t)-x_{j}\left(t^{\prime}\right)\right\|<\epsilon, \quad j=1,2, \ldots, m ; t, t^{\prime} \in J_{k}^{(i)}\left(k=0,1, \ldots, p ; i=1,2, \ldots, n_{k}\right)
$$

we have

$$
S(J)=\cup\left\{S_{j}\left(J_{k}^{(i)}\right): i=1,2, \ldots, n_{k} ; k=0,1, \ldots, p ; j=1,2, \ldots, m\right\}
$$


For $x(t), \bar{x}\left(t^{\prime}\right) \in S_{j}\left(J_{k}^{(i)}\right)$ (i.e., $\left.x, \bar{x} \in S_{j}, t, t^{\prime} \in J_{k}^{(i)}\right)$, we find by (17) and (15)

$$
\begin{gathered}
\left\|x(t)-\bar{x}\left(t^{\prime}\right)\right\| \leq\left\|x(t)-x_{j}(t)\right\|+\left\|x_{j}(t)-x_{j}\left(t^{\prime}\right)\right\|+\left\|x_{j}\left(t^{\prime}\right)-\bar{x}\left(t^{\prime}\right)\right\| \\
\leq\left\|x-x_{j}\right\|_{p}+\epsilon+\left\|x_{j}-x\right\|_{p} \\
<2 \operatorname{diam}\left(S_{j}\right)+\epsilon<2 \alpha(S)+3 \epsilon
\end{gathered}
$$

which implies

$$
\alpha(S(J)) \leq 2 \alpha(S)+3 \epsilon
$$

Since $\epsilon$ is arbitrary, it follows from (16) and (18) that

$$
\alpha\left(S\left(t_{k}\right)\right) \leq \alpha(S), k=1,2, \ldots, p
$$

and

$$
\alpha(S(J)) \leq 2 \alpha(S)
$$

Finally, (13), (14), (19) and (20) imply $\alpha(A(S)) \leq k_{r} \alpha(S)$, where $k_{r}=2 T L_{r}+\sum_{k=1}^{p} M_{r}^{(k)}<1$, and the lemma is proved.

We also need the following result which is concerned with the fixed points of strict set contractions (see [1], [2]):

Lemma 3: Let $K$ be a cone of the real Banach space $X$ and $K_{r, R}=\{x \in K$ : $r \leq\|x\| \leq R\}$ with $R>r>0$. Suppose that $B: K_{r, R \rightarrow K}$ is a strict set contraction such that one of the following two conditions is satisfied:

a) $B x \not x$ for $x \in K,\|x\|=r$ and $B x \geq x$ for $x \in K,\|x\|=R$.

b) $B x \geq x$ for $x \in K,\|x\|=r$ and $B x \not x$ for $x \in K,\|x\|=R$.

Then $B$ has at least one fixed point in $K_{r, R}$.

\section{MAIN THEOREMS}

Let us list some conditions for convenience.

$\left(H_{1}\right): H \in C[J \times J \times P, P], H(t, s, \theta)=\theta$ for $t, s \in J, I_{k} \in C[P, P], I_{k}(\theta)=\theta(k=1,2, \ldots, p)$.

For any $r>0, H$ is uniformly continuous on $J \times J \times\left(P \cap T_{r}\right), I_{k}$ is bounded on $P \cap T_{r}$ and there exist nonnegative constants $L_{r}$ and $M_{r}{ }^{(k)}$ with

$$
2 T L_{r}+\sum_{k=1}^{p} M_{r}^{(k)}<1
$$

such that (10) and (11) hold. There exist also $t_{p}<a<b<T$ and $0<c<1$ such that 


$$
H(t, s, x) \geq c H(u, s, x), t \in J_{0}=[a, b], u, s \in J, x \in P .
$$

$\left(H_{2}\right):\|H(t, s, x)\| /\|x\| \rightarrow 0$ as $x \in P$ and $\|x\| \rightarrow 0$ uniformly in $t, s \in J ;\left\|I_{k}(x)\right\| /\|x\| \rightarrow 0$ as $x \in P$ and $\|x\| \rightarrow 0(k=1,2, \ldots, p)$.

$\left(H_{3}\right) \quad\|H(t, s, x)\| /\|x\| \rightarrow 0$ as $x \in P$ and $\|x\| \rightarrow \infty$ uniformly in $t, s \in J ;\left\|I_{k}(x)\right\| /\|x\| \rightarrow 0$ as $x \in P$ and $\|x\| \rightarrow \infty(k=1,2, \ldots, p)$.

$\left(H_{4}\right)$ : there exists a $g \in P^{*}\left(P^{*}\right.$ denotes the dual cone of $\left.P\right)$ such that $g(x)>0$ for any $x>\theta$ and $g(H(t, s, x)) / g(x) \rightarrow \infty$ as $x \in P$ and $\|x\| \rightarrow 0$ uniformly in $t, s \in J_{0}$.

$\left(H_{5}\right)$ : there exists a $g \in P^{*}$ such that $g(x)>0$ for any $x>\theta$ and $g(H(t, s, x)) / g(x) \rightarrow \infty$ as $x \in P$ and $\|x\| \rightarrow \infty$ uniformly in $t, s \in J_{0}$.

Theorem 1: Let cone $P$ be normal. Suppose that conditions $\left(H_{1}\right),\left(H_{2}\right)$ and $\left(H_{5}\right)$ are satisfied. Then, equation (1) has at least one positive solution.

Proof: Let $K=\left\{x \in Q: x(t) \geq c x(s)\right.$ for $\left.t \in J_{0}, s \in J\right\}$. Then $K$ is a cone of $P C[J, E]$ and $K \subset Q$. For any $x \in Q$, we have by (21): $t \in J_{0}$ and $u \in J$ simply

$$
\begin{gathered}
A x(t)=\int_{0}^{T} H(t, s, x(s)) d s+\sum_{0<t_{k}<t} I_{k}\left(x\left(t_{k}\right)\right) \\
\geq c \int_{0}^{T} H(u, s, x(s)) d s+\sum_{0<t_{k}<t} I_{k}\left(x\left(t_{k}\right)\right) \\
=c \int_{0}^{T} H(u, s, x(s)) d s+\sum_{k=1}^{p} I_{k}\left(x\left(t_{k}\right)\right)\left(\text { since } a>t_{p}\right) \\
\geq c\left\{\int_{0}^{T} H(u, s, x(s)) d s+\sum_{0<t_{k}<u} I_{k}\left(x\left(t_{k}\right)\right)\right\}=c A x(u),
\end{gathered}
$$

hence $A x \in K$, and so

$$
A(K) \subset K
$$

Choose

$$
M>(b-a)^{-1}
$$

By $\left(H_{5}\right)$, there exists $h>0$ such that

$$
g(H(t, s, x)) \geq M g(x), t, s \in J_{0}, \quad x \in P,\|x\| \geq h .
$$

Now, for any 


$$
R>N h c^{-1}(N \text {-normal constant of } P),
$$

we are going to show that

$$
A x \not x \text { for } x \in K,\|x\|_{p}=R .
$$

In fact, if there exists $x_{0} \in K$ with $\left\|x_{0}\right\|_{p}=R$ such that $A x_{0} \leq x_{0}$, then $x_{0}(t) \geq c x_{0}(s)$, and so

$$
N\left\|x_{0}(t)\right\| \geq c\left\|x_{0}(s)\right\|, t \in J_{0}, s \in J
$$

which implies by (25)

$$
\left\|x_{0}(t)\right\| \geq c N^{-1}\left\|x_{0}\right\|_{p}=c N^{-1} R>h, t \in J_{0} .
$$

Also, we have

It follows from (28), (27) and (24) that

$$
x_{0}(t) \geq A x_{0}(t) \geq \int_{a}^{b} H\left(t, s, x_{0}(s)\right) d s, t \in J_{0} .
$$

$$
g\left(x_{0}(t)\right) \geq \int_{a}^{b} g\left(H\left(t, s, x_{0}(s)\right) d s \geq M \int_{a}^{b} g\left(x_{0}(s)\right) d s, t \in J_{0},\right.
$$

and so

$$
\int_{a}^{b} g\left(x_{0}(t)\right) d t \geq M(b-a) \int_{a}^{b} g\left(x_{0}(s)\right) d s .
$$

It is easy to see that

$$
\int_{a}^{b} g\left(x_{0}(t)\right) d t>0 \text {. }
$$

In fact, if this integral equals to zero, then $g\left(x_{0}(t)\right)=0$, and so $x_{0}(t)=\theta$ for any $t \in J_{0}$, which implies by virtue of $x_{0} \in K$ that $x_{0}(s)=\theta$ for $s \in J$, in contradiction with $\left\|x_{0}\right\|_{p}=R$. Now, (29) and (30) imply $M(b-a) \leq 1$, which contradicts (23), and therefore (26) is true.

On the other hand, on account of $\left(H_{2}\right)$ and $H(t, s, \theta)=\theta, I_{k}(\theta)=\theta$, we can find a $r>0(r<R)$ such that

$$
\|H(t, s, x)\| \leq m\|x\|, x \in P,\|x\| \leq r ; t, s \in J
$$

and

$$
\left\|I_{k}(x)\right\| \leq m\|x\|, x \in P,\|x\| \leq r ; k=1,2, \ldots, p
$$

where

$$
m=[2 N(T+p)]^{-1} .
$$

Now, we verify that 


$$
A x \nsupseteq x \text { for } x \in K,\|x\|_{p}=r .
$$

In fact, if there is $x_{1} \in K,\left\|x_{1}\right\|_{p}=r$ such that $A x_{1} \geq x_{1}$, then

$$
\theta \leq x_{1}(t) \leq \int_{0}^{T} H\left(t, s, x_{1}(s)\right) d s+\sum_{0<t_{k}<t} I_{k}\left(x_{1}\left(t_{k}\right)\right), t \in J,
$$

and so, by (31) and (32)

$$
\begin{aligned}
\left\|x_{1}(t)\right\| & \leq N m\left(\int_{0}^{T}\left\|x_{1}(s)\right\| d s+\sum_{k=1}^{p}\left\|x_{1}\left(t_{k}\right)\right\|\right) \\
& \leq N m(T+p)\left\|x_{1}\right\|_{p}, t \in J
\end{aligned}
$$

hence,

$$
r=\left\|x_{1}\right\|_{p} \leq N m(T+p)\left\|x_{1}\right\|_{p}=N m(T+p) r,
$$

which contradicts (33), and therefore (34) is true.

By Lemma 2, $A$ is a strict set contraction on $K_{r, R}=\left\{x \in K: r \leq\|x\|_{p} \leq R\right\}$. Observing (22), (26) and (34) and using Lemma 3, we see that $A$ has a fixed point in $K_{r, R}$, which is a positive solution of (1). The proof is complete.

Theorem 2: Let cone $P$ be normal. Suppose that conditions $\left(H_{1}\right),\left(H_{3}\right)$ and $\left(H_{4}\right)$ are satisfied. Then, equation (1) has at least one positive solution.

Proof: The proof is similar. First, (22) holds. In the same way as establishing (26) we can show: $\left(H_{4}\right)$ implies that there exists $r>0$ such that

$$
A x \not x \text { for } x \in K,\|x\|_{p}=r .
$$

On the other hand, by $\left(\mathrm{H}_{3}\right)$ there is a $q>0$ such that

$$
\|H(t, s, x)\| \leq m\|x\|, x \in P,\|x\| \geq q, t, s \in J
$$

and

$$
\left\|I_{k}(x)\right\| \leq m\|x\|, x \in P,\|x\| \geq q ; k=1,2, \ldots, p
$$

where

$$
m=[2 N(T+p)]^{-1}
$$

Consequently,

and

$$
\|H(t, s, x)\| \leq m\|x\|+M, x \in P, t, s \in J
$$




$$
\left\|I_{k}(x)\right\| \leq m\|x\|+M, x \in P
$$

where, by $\left(H_{1}\right)$,

$$
M=\max \left\{\sup _{J \times J \times\left(P \cap T_{q}\right)}\|H(t, s, x)\|, \sup _{P \cap T_{q}}\left\|I_{k}(x)\right\|, k=1,2, \ldots, p\right\}<\infty .
$$

Choose $R>\max \{r, 2 N M(T+p)\}$. Then, it is easy to show as establishing (34) that

$$
A x \geq x \text { for } x \in K,\|x\|_{p}=R .
$$

Hence, again Lemma 3 implies that $A$ has a fixed point in $K_{r, R}$.

Remark 1: In particular case of one dimensional space, $E=R, P=R_{+}$and $P^{*}=P=R_{+}$. In this case, (10) and (11) are satisfied automatically and we may choose $g=1$ in $\left(H_{4}\right)$ and $\left(H_{5}\right)$. Hence, from Theorems 1 and 2 , we get the following

Conclusion: Let $H \in C\left[J \times J \times R_{+}, R_{+}\right], H(t, s, 0)=0, I_{k} \in C\left[R_{+}, R_{+}\right], I_{k}(0)=0$ $(k=1,2, \ldots, p)$ and there exist $t_{p}<a<b<T$ and $0<c<1$ such that

$$
H(t, s, x) \geq c H(u, s, x), t \in J_{0}=[a, b], u, s \in J, x \geq 0 .
$$

Suppose that one of the following two conditions is satisfied:

a) $\quad H(t, s, x) / x \rightarrow 0$ as $x \rightarrow+0$ uniformly in $t, s \in J ; \quad I_{k}(x) / x \rightarrow 0$ as $x \rightarrow+0$ $(k=1,2, \ldots, p)$,

and $H(t, s, x) / x \rightarrow+\infty$ as $x \rightarrow+\infty$ uniformly in $t, s \in J_{0}$.

b) $\quad H(t, s, x) / x \rightarrow 0$ as $x \rightarrow+\infty$ uniformly in $t, s \in J ; I_{k}(x) / x \rightarrow 0$ as $x \rightarrow+\infty$

$(k=1,2, \ldots, p)$, and $H(t, s, x) / x \rightarrow+\infty$ as $x \rightarrow+0$ uniformly in $t, s \in J_{0}$.

Then, equation (1) has at least one positive solution.

\section{APPLICATIONS}

Consider the two-point boundary value problem for second order impulsive differential equation in $E$ :

$$
\left\{\begin{array}{c}
-x^{\prime \prime}=f(t, x), t \neq t_{k}(k=1,2, \ldots, p) \\
\left.\Delta x\right|_{t=t_{k}}=I_{k}(x),(k=1,2, \ldots, p) \\
x(0)=x^{\prime}(1)=\theta
\end{array}\right.
$$

where $0<t_{1}<\ldots<t_{k}<\ldots<t_{p}<1, f \in C[J \times P, P], \quad J=[0,1], \quad f(t, \theta)=\theta, \quad I_{k} \in C[P, P]$, $I_{k}(\theta)=\theta \quad(k=1,2, \ldots, p)$. Evidently, $x(t) \equiv \theta$ is the trivial solution of (37). Let $J^{\prime}$ $=J \backslash\left\{t_{1}, t_{2}, \ldots, t_{p}\right\}$. A map $x \in P C[J, E] \cap C^{2}\left[J^{\prime}, E\right]$ is called a positive solution of (37) if it satisfies (37) and $x \in Q, x \neq \theta(Q$ is defined as before, i.e., $Q=\{x \in P C[J, E]: x(t) \geq \theta$ for 
$t \in J\})$.

We list the following conditions:

$\left(H_{1}^{\prime}\right): f \in C[J \times P, P], J=[0,1], f(t, \theta)=\theta$ for $t \in J, I_{k} \in C[P, P], I_{k}(\theta)=\theta(k=1,2, \ldots, p)$.

For any $r>0, f$ is uniformly continuous on $J \times\left(P \cap T_{r}\right), I_{k}$ is bounded on $P \cap T_{r}$, and there exist nonnegative constants $L_{r}$ and $M_{r}{ }^{(k)}$ with

$$
2 T L_{r}+\sum_{k=1}^{p} M_{r}^{(k)}<1
$$

such that

$$
\alpha(f(t, D)) \leq L_{r} \alpha(D), t \in J, D \subset P \cap T_{r}
$$

and

$$
\alpha\left(I_{k}(D)\right) \leq M_{r}^{(k)} \alpha(D), D \subset P \cap T_{r}, k=1,2, \ldots, p
$$

$\left(H_{2}^{\prime}\right):\|f(t, x)\| /\|x\| \rightarrow 0$ as $x \in P$ and $\|x\| \rightarrow 0$ uniformly in $t \in J ;\left\|I_{k}(x)\right\| /\|x\| \rightarrow 0$ as $x \in P$ and $\|x\| \rightarrow 0(k=1,2, \ldots, p)$.

$\left(H_{3}^{\prime}\right):\|f(t, x)\| /\|x\| \rightarrow 0$ as $x \in P$ and $\|x\| \rightarrow \infty$ uniformly in $t \in J ;\left\|I_{k}(x)\right\| /\|x\| \rightarrow 0$ as $x \in P$ and $\|x\| \rightarrow \infty(k=1,2, \ldots p)$.

$\left(H_{4}^{\prime}\right)$ : there exists $t_{p}<a<b<1$ and $g \in P^{*}$ such that $g(x)>0$ for any $x>\theta$ and $g(f(t, x)) / g(x) \rightarrow \infty$ as $x \in P$ and $\|x\| \rightarrow 0$ uniformly in $t \in J_{0}=[a, b]$.

$\left(H_{5}^{\prime}\right)$ : there exists $t_{p}<a<b<1$ and $g \in P^{*}$ such that $g(x)>0$ for any $x>\theta$ and $g(f(t, x)) / g(x) \rightarrow \infty$ as $x \in P$ and $\|x\| \rightarrow \infty$ uniformly in $t \in J_{0}=[a, b]$.

Theorem 3: Let cone $P$ be normal. Suppose that conditions $\left(H_{1}^{\prime}\right),\left(H_{2}^{\prime}\right)$ and $\left(H_{5}^{\prime}\right)$ are satisfied. Then, BVP (37) has at least one positive solution.

Theorem 4: Let cone $P$ be normal. Suppose that conditions $\left(H_{1}^{\prime}\right),\left(H_{3}^{\prime}\right)$ and $\left(H_{4}^{\prime}\right)$ are satisfied. Then, BVP (37) has at least one positive solution.

Proof of Theorems 3 and 4: It is easy to see that $x \in P C[J, E] \cap C^{2}\left[J^{\prime}, E\right]$ is a positive solution of (37) if and only if $x \in P C[J, E]$ is a positive solution of the following impulsive integral equation:

$$
x(t)=\int_{0}^{1} G(t, s) f(s, x(s)) d s+\sum_{0<t_{k}<t} I_{k}\left(x\left(t_{k}\right)\right),
$$

where $G(t, s)$ is the Green function for the differential operator $-x^{\prime \prime}$ under boundary condition $x(0)=x^{\prime}(1)=0$, i.e., 


$$
G(t, s)=\min \{t, s\}=\left\{\begin{array}{c}
t, t \leq s \\
s, t>s .
\end{array}\right.
$$

Evidently, (38) is an equation of the form (1) with $T=1$ and $H(t, s, x)=G(t, s) f(s, x)$. It is easy to show that $0 \leq G(t, s) \leq 1(t, s \in J=[0,1])$, and for any $t_{p}<a<b<1$,

$$
G(t, s) \geq a G(u, s), t \in[a, b], u, s \in J .
$$

Hence, (21) is satisfied for any $t_{p}<a<b<1$ with $c=a$. Consequently, it is clear that $\left(H_{1}^{\prime}\right)$, $\left(H_{2}^{\prime}\right)$ and $\left(H_{5}^{\prime}\right)$ imply $\left(H_{1}\right),\left(H_{2}\right)$ and $\left(H_{5}\right)$, and $\left(H_{1}^{\prime}\right),\left(H_{3}^{\prime}\right)$ and $\left(H_{4}^{\prime}\right)$ imply $\left(H_{1}\right),\left(H_{3}\right)$ and $\left(H_{4}\right)$. Thus, Theorems 3 and 4 follow from Theorems 1 and 2 respectively.

Remark 2: In one dimensional case, $E=R, P=P^{*}=R_{+}$and $g=1$, we get the following result from Theorems 3 and 4 :

Conclusion: Let $f \in C\left[J \times R_{+}, R_{+}\right], \quad J=[0,1], \quad f(t, 0)=0, \quad I_{k} \in C\left[R_{+}, R_{+}\right]$, $I_{k}(0)=0(k=1,2, \ldots, p)$. Suppose that one of the following two conditions is satisfied:

$\left.a^{\prime}\right) \quad f(t, x) / x \rightarrow 0$ as $x \rightarrow+0$ uniformly in $t \in J, I_{k}(x) / x \rightarrow 0$ as $x \rightarrow+0(k=1,2, \ldots, p)$ and there exist $t_{p}<a<b<1$ such that $f(t, x) / x \rightarrow+\infty$ as $x \rightarrow+\infty$ uniformly in $t \in[a, b]$.

$\left.b^{\prime}\right) \quad f(t, x) / x \rightarrow 0$ as $x \rightarrow+\infty$ uniformly in $t \in J, I_{k}(x) / x \rightarrow 0$ as $x \rightarrow+\infty(k=1,2, \ldots, p)$ and there exist $t_{p}<a<b<1$ such that $f(t, x) / x \rightarrow+\infty$ as $x \rightarrow+0$ uniformly in $t \in[a, b]$.

Then, $B V P(37)$ has at least one positive solution. 


\section{REFERENCES}

[1] A.J.B. Botter, "A fixed point theorem for positive $k$-set contractions", Proc. Edinburgh Math. Soc., 19 (2), (1974), pp. 93-102.

[2] N.P. Cac and J.A. Gatica, "Fixed point theorems for mappings in ordered Banach spaces”, J. Math. Anal. Appl., 71, (1979), pp. 547-557.

[3] L.H. Erbe and X. Liu, "Quasisolutions of nonlinear impulsive equations in abstract cones", Appl. Anal., 34, (1989), pp. 231-250.

[4] D. Guo and V. Lakshmikantham, "Nonlinear Problems in Abstract Cones", Academic Press, Inc., Boston and New York, 1988.

[5] V. Lakshmikantham, "Trends in the theory of impulsive differential equations", Differential Equations and Applications, Vol. I, II, (1989), pp. 76-87.

[6] V. Lakshmikantham and S. Leela, "Nonlinear Differential Equations in Abstract Spaces", Pergamon Press, Oxford, England, 1981. 


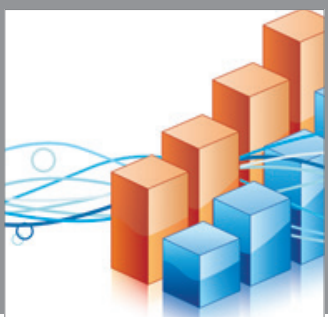

Advances in

Operations Research

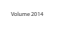

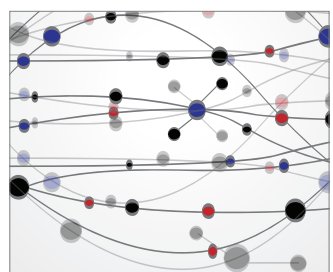

\section{The Scientific} World Journal
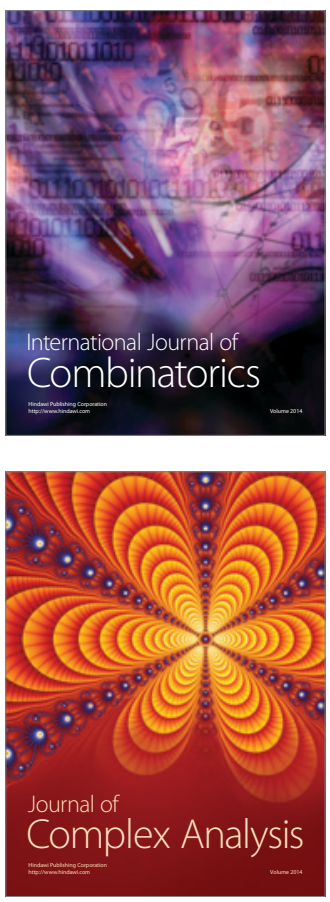

International Journal of

Mathematics and

Mathematical

Sciences
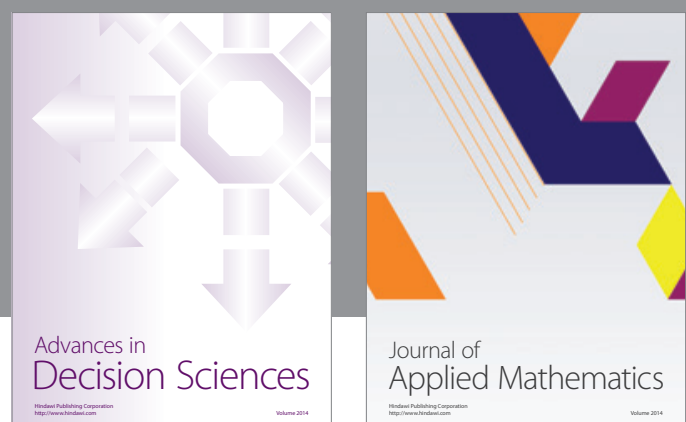

Journal of

Applied Mathematics
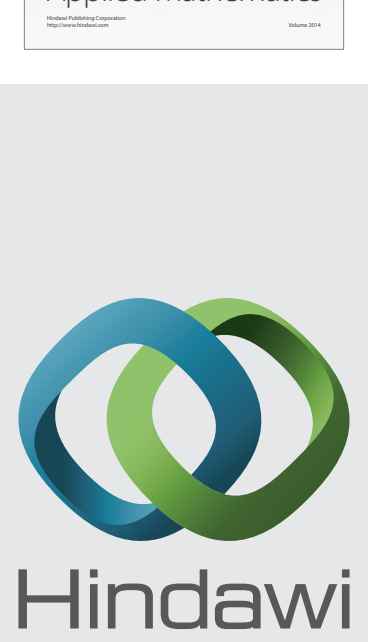

Submit your manuscripts at http://www.hindawi.com
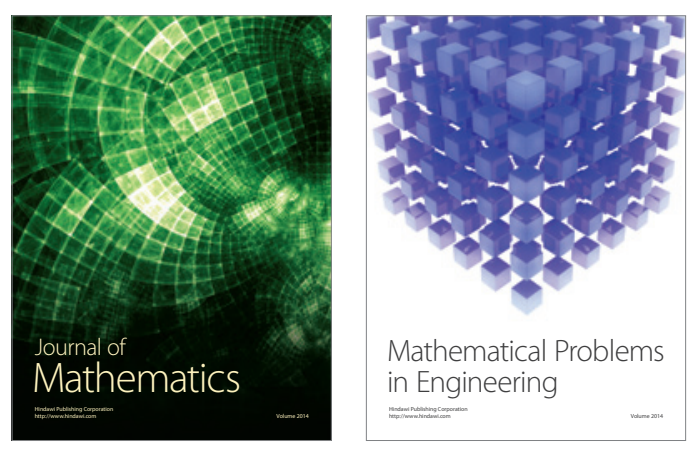

Mathematical Problems in Engineering
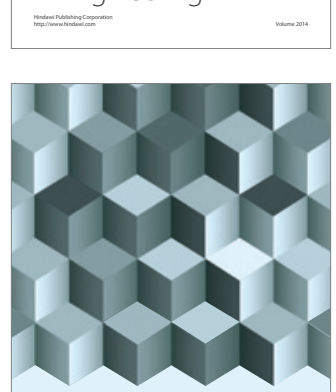

Journal of

Function Spaces
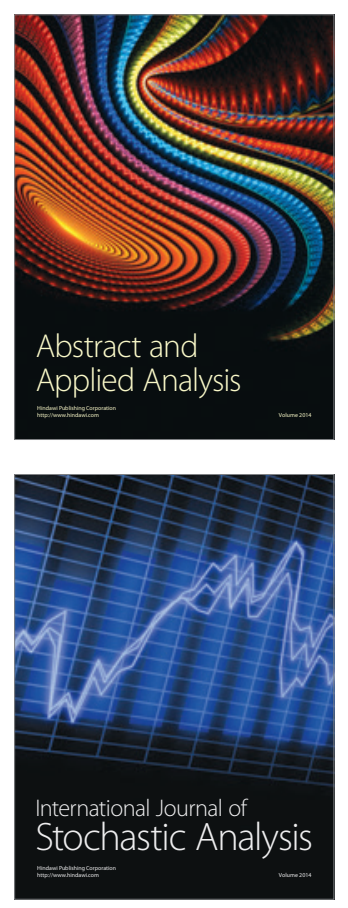

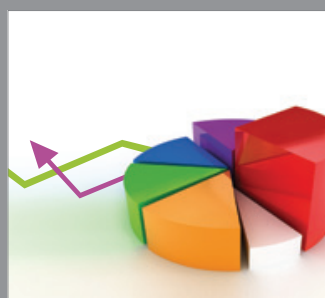

ournal of

Probability and Statistics

Promensencen
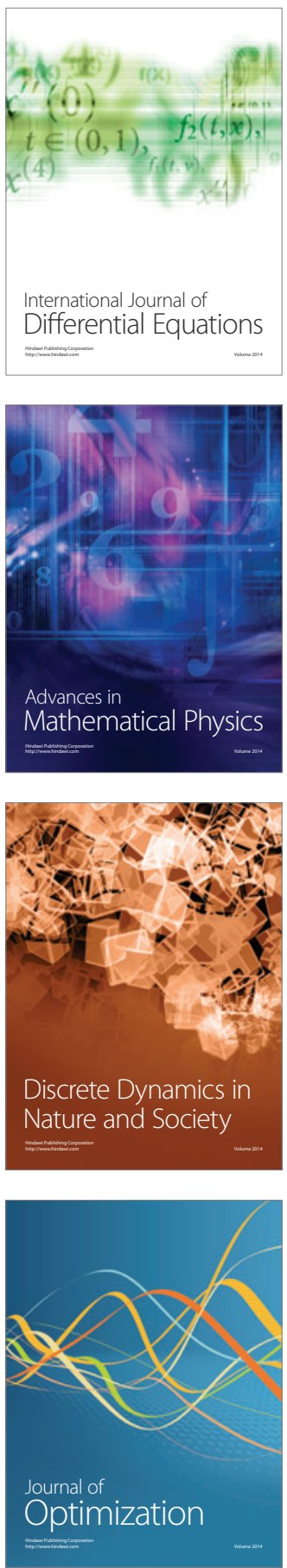